ENSEÑANZA DE LA QUÍMICA

\title{
ESTUDIO ESPECTROSCÓPICO RMN BIDIMENSIONAL DE BASES DE SCHIFF DERIVADAS DE LA 4-AMINOANTIPIRINA
}

\author{
Sergio Zamorano $^{1 *}$, Juan Camus ${ }^{1}$, Mariana Zavala ${ }^{1}$
}

\begin{abstract}
RESUMEN
Se ha realizado el análisis estructural de seis derivados de la 4-aminoantipirina (4AAP) mediante dos técnicas de RMN bidimensional, la HSQC y la HMBC, las que ayudaron a dilucidar la estructura de los cuatro derivados utilizando los datos suministrados por la interacción entre los átomos de carbono e hidrógeno presentes en las diferentes moléculas.
\end{abstract}

Palabras clave: derivados de la 4- aminoantipirina, espectroscopía RMN 2D

\section{TTWO-DIMENSIONAL NMR SPECTROSCOPIC STUDY OF SCHIFF BASES DERIVED FROM 4-AMINOANTIPYRINE}

\begin{abstract}
There has been the structural analysis of six derivatives of the 4- aminoantipyrine(4AAP) by means of two techniques of two-dimensional NMR, the HSQC and HMBC, which helped to elucidate the structure of four derivatives using the data provided by the interaction between the atoms of carbon and hydrogen present in the different molecules.
\end{abstract}

Key words: 4-aminoantipyrine derivatives, 2D NMR spectroscopy

\section{INTRODUCCIÓN}

En trabajos anteriores hemos determinado la estructura de bases de Schiff derivadas del 2aminofenol ${ }^{1,2}$ utilizando técnicas de RMN 1D y 2D. Numerosos investigadores han sintetizado bases de Schiff hexadentadas, que podrían utilizarse en la síntesis de numerosos compuestos complejos que presentan actividad óptica ${ }^{3,4}$, y también para compuestos complejos tetradentados transportadores de oxígeno de tipo Co-Salen y sus derivados sustituidos en el ciclo, que tienen propiedades de fijar el oxígeno cuando se encuentran en estado sólido ${ }^{5}$. Las bases de Schiff (iminas) son, en general, el producto de condensación del amoniaco o una

\footnotetext{
${ }^{1}$ Facultad de Ciencias Naturales y Exactas. Carvallo N²70. Universidad de Playa Ancha. Valparaíso. Chile *szamoran@upla.cl
} 
amina primaria con una cetona o un aldehído. En nuestro caso hemos utilizado la reacción de condensación de la 4-amino antipirina (4AAP) con diversos aldehídos (2-metilbenzaldehido ${ }^{6}$, p-nitrobenzaldehído ${ }^{7}, 2$-hidroxibenzaldehído ${ }^{8}, \mathrm{p}$-dimetilaminabenzaldehído ${ }^{9}$, 4-bromobenzaldehído, 2-nitrobenzaldehído). La reacción es reversible y se favorece en un medido ligeramente ácido, que permite la protonación del oxígeno del grupo carbonilo para favorecer el ataque nucleófilo ${ }^{10}$ por la amina de la 4AAP, lo que da como resultado la sustitución del enlace $\mathrm{C}=\mathrm{O}$ por $\mathrm{C}=\mathrm{N}$. Todos estos derivados de la 4 AAP estudiados en este trabajo, han sido sintetizados por diversos investigadores ${ }^{11-21}$ y se han ensayado para sintetizar compuestos de coordinación, actividad biológica (antiinflamatoria, antibacteriana, antifúngicos, anticancerígena, analgésico, antipirético), inhibidores de corrosión, sensores fluorescentes, etc., información que se encuentra muy bien recopilada en los reviews de Deshmukh et $a l^{22}$ y Raman et $a l^{23}$. La 4-aminoantipirina se utiliza para la determinación cuantitativa de fenoles, porque estos reaccionan con la 4AAP en medio alcalino en presencia de ferrocianuro de potasio generando un compuesto rosado, que permite la determinación fotométrica cuantitativa de los fenoles ${ }^{24,25}$. Sin embargo, los estudios de RMN bidimensionales de estos derivados no han sido publicados y constituyen un interesante aporte a la información estructural de estos compuestos.

La RMN bidimensional ha resultado ser una excelente técnica para dilucidar la estructura de las moléculas orgánicas, así por ejemplo, utilizando la técnica bidimensional heteronuclear HMBC permite la detección de protones, que muestra los carbonos que se encuentran a 2 o 3 enlaces de distancia de los protones y el experimento HSQC, que permite la detección de protones $\left({ }^{1} \mathrm{H}\right)$ que muestra los carbones $\left({ }^{13} \mathrm{C}\right)$ directamente conectados, en otras palabras, estos experimentos permiten observar los protones enlazados directamente o remotamente, hasta 4 enlaces $^{26,27}$. En este trabajo utilizaremos las técnicas HSQC y HMBC para dilucidar la estructura de seis diferentes bases de Schiff derivadas de la 4AAP.

\section{PARTE EXPERIMENTAL}

\section{Materiales y métodos}

La síntesis de las correspondientes bases de Schiff fue producto de la reacción de condensación entre la 4-aminoantipirina y los siguientes aldehídos:

4-bromobenzaldehído, 2-hidroxibenzaldehído, 2-metilbenzaldehído, 2-nitrobenzaldehído, 4-dimetilaminobenzaldehído y 4-nitrobenzaldehído.

El procedimiento consistió en disolver 1,2 mmol de la 4AAP en $100 \mathrm{ml}$ de etanol, en un balón de $200 \mathrm{ml}$ provisto de sistema de reflujo, al que se añadieron 1,2 mmol del aldehído correspondiente, en relación 1:1. Las soluciones resultantes se sometieron a reflujo durante una hora, bajo constante agitación. Terminado este proceso, las soluciones de color amarillo se mantuvieron bajo refrigeración durante una semana para obtener cristales aciculares de color amarillo. 


\section{RESULTADOS Y DISCUSIÓN}

La reacción entre la 4-aminoantipirina y el aldehído respectivo se produce de acuerdo a una reacción de condensación similar en los seis derivados utilizados; de manera que podemos generalizar, esquematizando las moléculas resultantes de acuerdo a la figura 1.<smiles>[R]c1ccc(C=O)c([R4])c1</smiles>

Figura 1. Reacción de condensación entre la 4AAP y los diversos aldehídos

Donde:

\begin{tabular}{|c|c|c|}
\hline $\mathrm{R}_{1}$ & $\mathrm{R}_{2}$ & $\begin{array}{c}\text { Formula } \\
\text { condensada BS }\end{array}$ \\
\hline$-\mathrm{H}$ & $-\mathrm{Br}$ & $\mathrm{C}_{18} \mathrm{H}_{16} \mathrm{BrN}_{3} \mathrm{O}$ \\
\hline$-\mathrm{OH}$ & $-\mathrm{H}$ & $\mathrm{C}_{18} \mathrm{H}_{17} \mathrm{~N}_{3} \mathrm{O}_{2}$ \\
\hline$-\mathrm{CH}_{3}$ & $-\mathrm{H}$ & $\mathrm{C}_{19} \mathrm{H}_{19} \mathrm{~N}_{3} \mathrm{O}$ \\
\hline$-\mathrm{H}$ & $-\mathrm{N}\left(\mathrm{CH}_{3}\right)_{2}$ & $\mathrm{C}_{20} \mathrm{H}_{22} \mathrm{~N}_{4} \mathrm{O}$ \\
\hline$-\mathrm{NO}_{2}$ & $-\mathrm{H}$ & $\mathrm{C}_{18} \mathrm{H}_{16} \mathrm{~N}_{4} \mathrm{O}_{3}$ \\
\hline$-\mathrm{H}$ & $-\mathrm{NO}_{2}$ & $\mathrm{C}_{18} \mathrm{H}_{16} \mathrm{~N}_{4} \mathrm{O}_{3}$ \\
\hline
\end{tabular}

En los espectros HSQC se obtienen las señales que entregan información acerca de la relación existente entre los núcleos de ${ }^{13} \mathrm{C}$ y ${ }^{1} \mathrm{H}$ que se encuentran unidos directamente.

En los espectros bidimensionales HMBC se obtienen las señales que entregan información acerca de la relación existente entre los núcleos de ${ }^{13} \mathrm{C}$ y ${ }^{1} \mathrm{H}$ que se encuentran a una distancia de 2 a 3 enlaces, y en casos especiales hasta 4 enlaces.

Con la información de los espectros HSQC y HMBC, y apoyados con técnicas de espectroscopia IR, se pueden determinar las estructuras de las seis bases de Schiff sintetizadas, siguiendo la metodología tradicional que se inicia marcando los carbonos, de manera correlativa con números del 1 al 20, según corresponda; y a los hidrógenos se les asignan letras minúsculas de la "a" a la "n", según corresponda.

La asignación de números y letras a los carbonos e hidrógenos de la base de Schiff 4AAP-Br se observa en la figura 2 . 


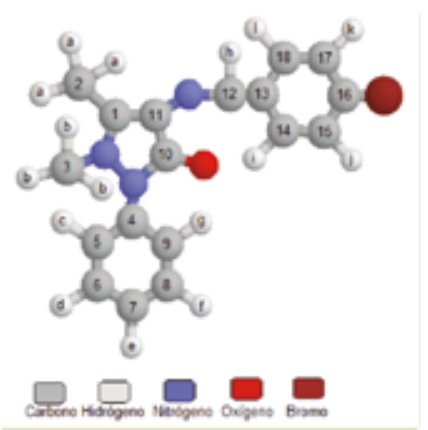

Figura 2. Asignación de números y letras a los carbonos e hidrógenos de la base de Schiff 4AAP-Br

La asignación de números y letras a los carbonos e hidrógenos de la base de Schiff 4AAPOH se observa en la figura 3.

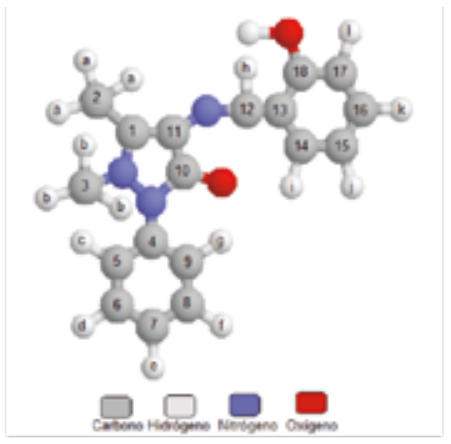

Figura 3. Asignación de números y letras a los carbonos e hidrógenos de la base de Schiff 4AAP-OH

La asignación de números y letras a los carbonos e hidrógenos de la base de Schiff 4AAP-Me se observa en la figura 4.

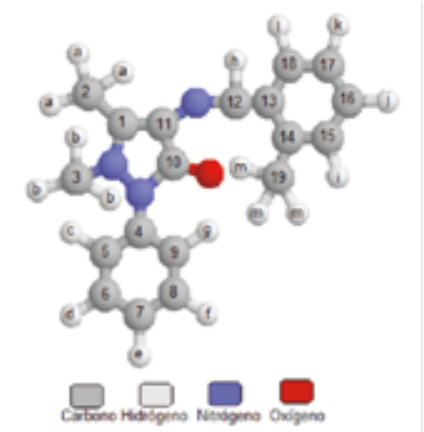

Figura 4. Asignación de números y letras a los carbonos e hidrógenos de la base de Schiff 4AAP-Me 
La asignación de números y letras a los carbonos e hidrógenos de la base de Schiff 4AAPDMA se observa en la figura 5 .

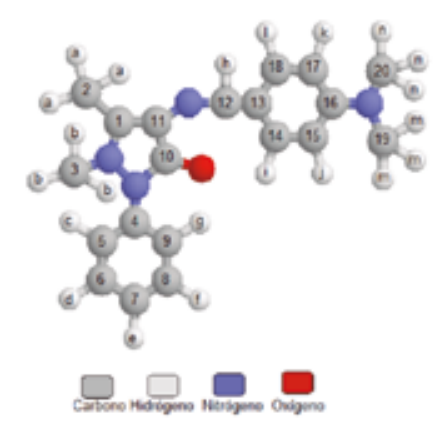

Figura 5. Asignación de números y letras a los carbonos e hidrógenos de la base de Schiff 4AAP-DMA

La asignación de números y letras a los carbonos e hidrógenos de la base de Schiff 4AAP2NO2 se observa en la figura 6 .

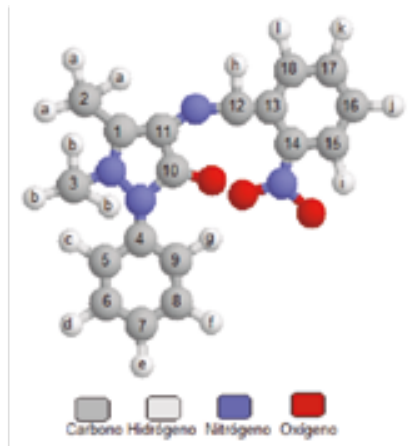

Figura 6. Asignación de números y letras a los carbonos e hidrógenos de la base de Schiff 4 AAP- $2 \mathrm{NO}_{2}$

La asignación de números y letras a los carbonos e hidrógenos de la base de Schiff 4AAP4NO2 se observa en la figura 7.

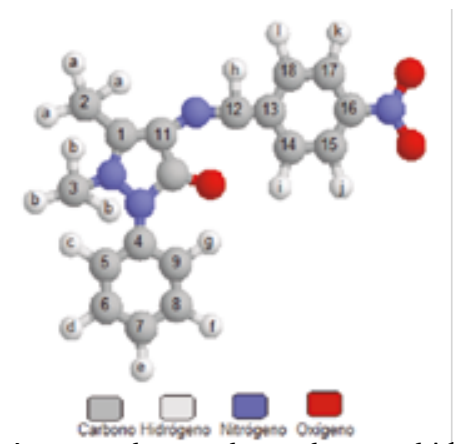

Figura 7. Asignación de números y letras a los carbonos e hidrógenos de la base de Schiff $4 \mathrm{AAP}-4 \mathrm{NO}_{2}$ 
Los espectros HMBC o también llamados "diagramas de contorno" de estas seis bases de Schiff, se presentan en las figuras 8-14. Estos espectros han permitido identificar cada uno de los carbonos y la conectividad que tienen con los protones que se encuentren a 2, 3 y 4 enlaces de distancia para poder determinar, en definitiva, la estructura de la molécula.

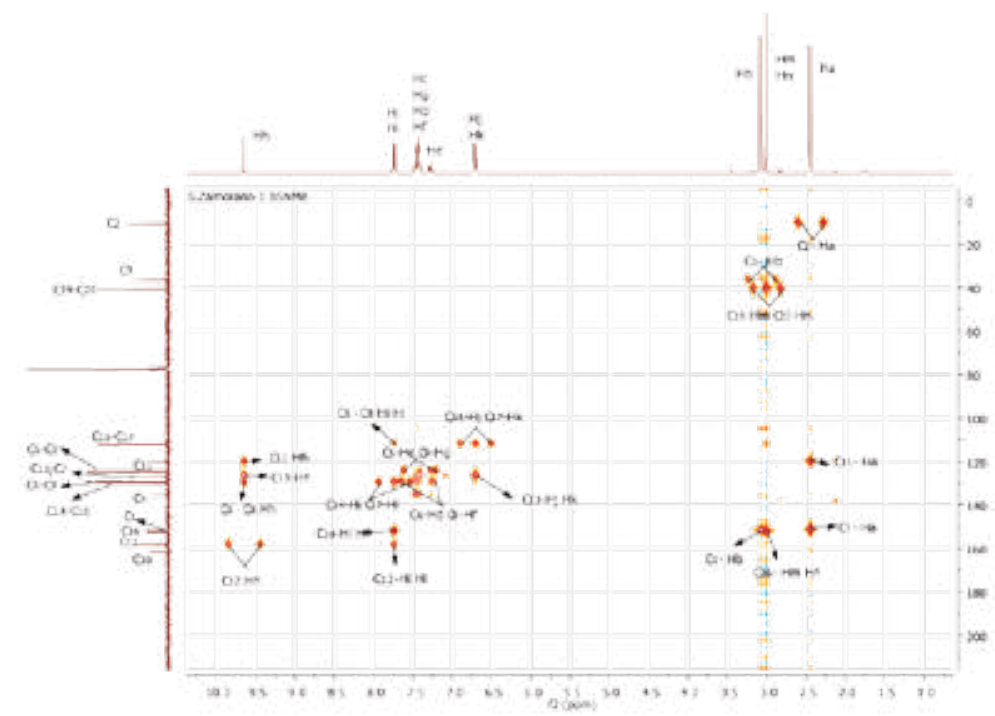

Figura 8. Espectro de RMN HMBC de la Base de Schiff 4AAP-DMA

En este diagrama es posible observar el acoplamiento de la señal del $\mathrm{C}_{2}$ con el $\mathrm{H}_{\mathrm{a}}$, y además

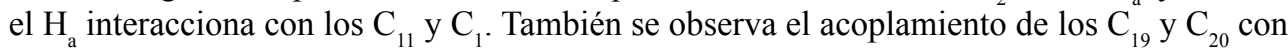
los $\mathrm{H}_{\mathrm{m}}$ y $\mathrm{H}_{\mathrm{n}}$, y la interacción de estos protones con el $\mathrm{C}_{16}$. Se observa otro acoplamiento del $\mathrm{C}_{12}$ con el $\mathrm{H}_{\mathrm{h}}$, y la interacción del $\mathrm{H}_{\mathrm{k}}$ con este carbono. Y además de otra interacción del $\mathrm{H}_{\mathrm{h}}$ con el $\mathrm{C}_{13}$ y $_{11}$.

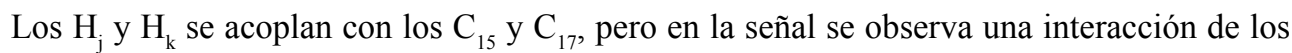
protones con el $\mathrm{C}_{16}$. Además otra interacción con el $\mathrm{C}_{12}$ con el $\mathrm{H}_{\mathrm{k}}$

El acoplamiento entre $\operatorname{los} \mathrm{C}_{14} \mathrm{y}_{18}$ con los $\mathrm{H}_{\mathrm{i}}$ y $\mathrm{H}_{1}$ se observa, y los protones de esta señal tienen una interacción con el $\mathrm{C}_{13}$.

Los $\mathrm{C}_{5}$ y $\mathrm{C}_{9}$ se acoplan con los $\mathrm{H}_{\mathrm{c}}$ y $\mathrm{H}_{\mathrm{g}}$, y los protones de esta señal interactúan con los $\mathrm{C}_{4}$ $\mathrm{y} \mathrm{C}_{7}$,

En la señal se observa otro acoplamiento entre los $\mathrm{C}_{6}$ y $\mathrm{C}_{8}$ con $\operatorname{los} \mathrm{H}_{\mathrm{d}}$ y $\mathrm{H}_{\mathrm{f}}$, y una interacción de los protones con el $\mathrm{C}_{7}$. 


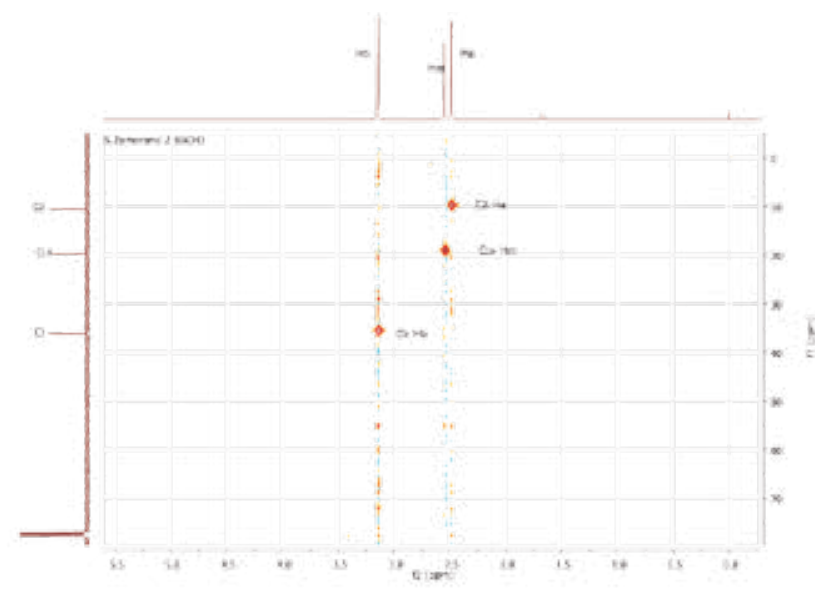

Figura 9. Espectro de RMN HMBC de la Base de Schiff 4AAP-M, para carbonos de los grupos metilo 2, 3, 19 y sus protones a, b y $\mathrm{m}$.

Este espectro muestra aquellas señales que corresponden a las interacciones entre $\operatorname{los} \mathrm{C}_{2}, \mathrm{C}_{3}$, $\mathrm{C}_{19}$ y los protones $\mathrm{H}_{\mathrm{a}}, \mathrm{H}_{\mathrm{b}} \mathrm{y} \mathrm{H}_{\mathrm{m}}$, respectivamente.

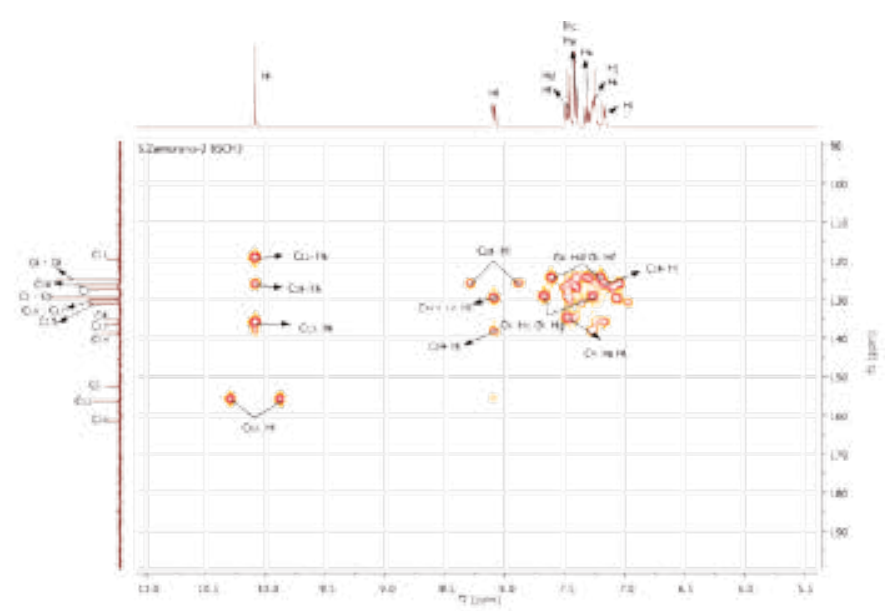

Figura 10. Espectro de RMN HMBC de la Base de Schiff 4AAP-M para la zona aromática.

Este espectro nos muestra la interacción del $\mathrm{C}_{11}$ cuaternario con el Hh a tres enlaces. $\mathrm{El} \mathrm{C}_{18}$ está unido directamente con el $\mathrm{H}_{1}$, interacciona con el $\mathrm{H}_{\mathrm{i}}$ y $\mathrm{H}_{\mathrm{h}}$ a $\mathbf{3}$ y $\mathbf{2}$ enlaces, respectivamente. El $\mathrm{C}_{12}$ está unido con el $\mathrm{H}_{\mathrm{h}}$, e interacciona con los protones a tres enlaces. 


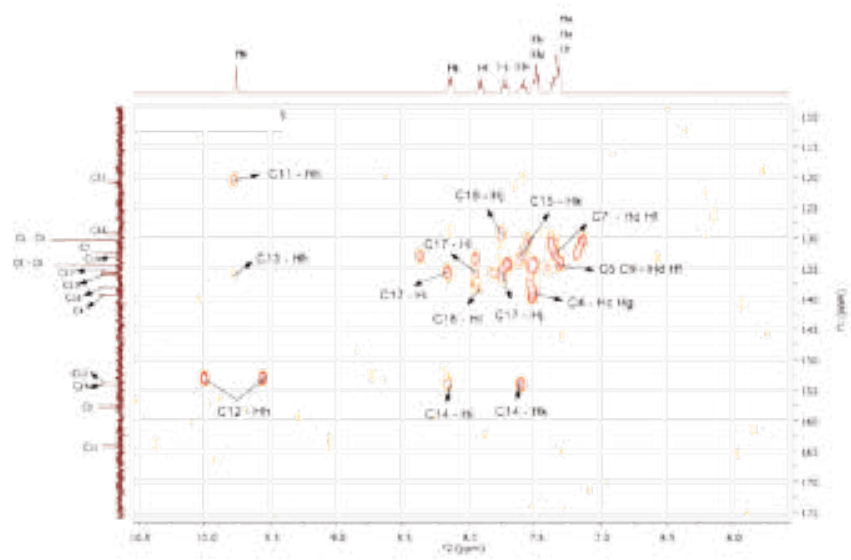

Figura 11. Espectro de RMN HMBC de la Base de Schiff $4 \mathrm{AAP}-2 \mathrm{NO}_{2}$ para la zona aromática.

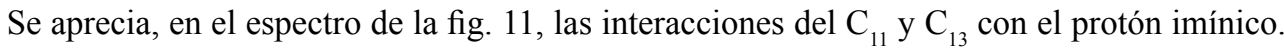
A su vez, se observa la interacción del $\mathrm{C}_{14}$ con el $\mathrm{H}_{\mathrm{i}}$, y la interacción del $\mathrm{C}_{4}$ con los $\mathrm{H}_{\mathrm{c}} \mathrm{y} \mathrm{H}_{\mathrm{g}}$. También se aprecia las interacciones del $\mathrm{C}_{17}$ con los $\mathrm{H}_{\mathrm{i}} \mathrm{y} \mathrm{H}_{1} \mathrm{y} \mathrm{H}_{\mathrm{j}}$; del $\mathrm{C} 16$ con el $\mathrm{H}_{\mathrm{l}}$; del $\mathrm{C}_{18}$ con el $\mathrm{H}_{\mathrm{j}}$; $\mathrm{y}$ del $\mathrm{C}_{15}$ con el $\mathrm{H}_{\mathrm{k}}$.

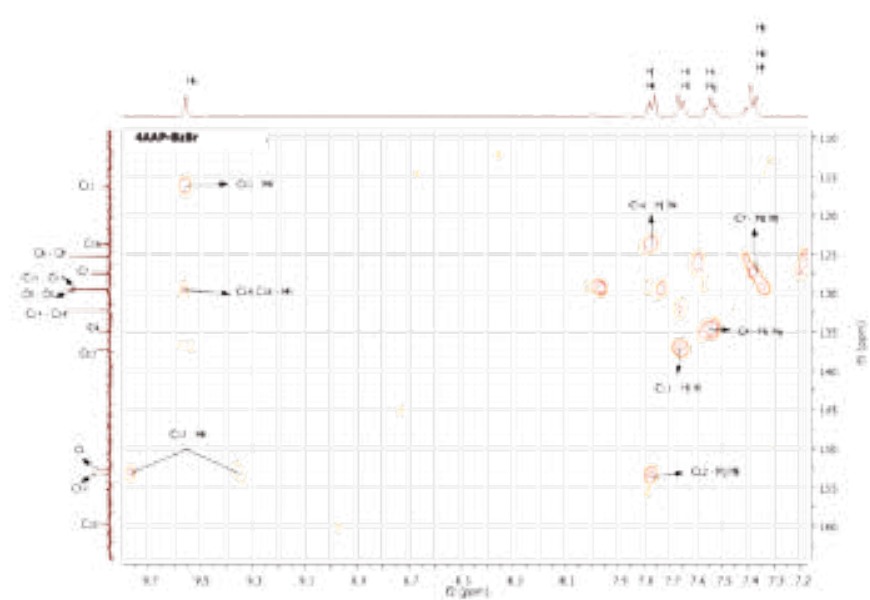

Figura 12. Espectro de RMN HMBC de la Base de Schiff 4AAP-Br para la zona aromática.

En el espectro se observa la interacción de los $\mathrm{C}_{11}, \mathrm{C}_{14} \mathrm{y} \mathrm{C}_{18}$ con el protón imínico. También se aprecia la interacción de los carbonos cuaternarios $\mathrm{C}_{16}, \mathrm{C}_{13} \mathrm{y} \mathrm{C}_{4}$ con los $\mathrm{H}_{\mathrm{j}} \mathrm{y} \mathrm{H}_{\mathrm{k}}$, con los $\mathrm{Hi}$ y $\mathrm{H}_{\mathrm{i}}$; y con los $\mathrm{H}_{\mathrm{c}}$ y $\mathrm{H}_{\mathrm{g}}$, respectivamente. 


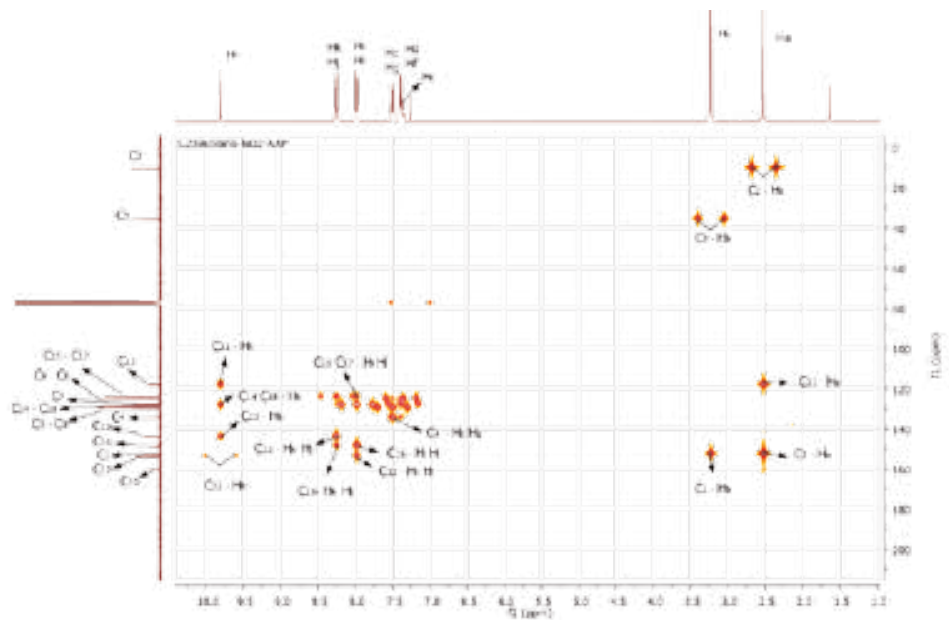

Figura 13. Espectro de RMN HMBC de la Base de Schiff $4 \mathrm{AAP}-4 \mathrm{NO}_{2}$

Este espectro muestra la interacción de los $\mathrm{C}_{11}, \mathrm{C}_{14}, \mathrm{C}_{18}$ y $\mathrm{C}_{13}$ con el protón imínico; la interacción del $\mathrm{C}_{16}$ con los $\mathrm{H}_{\mathrm{k}}, \mathrm{H}_{\mathrm{j}}$, Hi y $\mathrm{H}_{\mathrm{j}}$; la interacción del $\mathrm{C}_{13}$ con los $\mathrm{H}_{\mathrm{k}} \mathrm{y} \mathrm{H}_{\mathrm{j}}$; la interacción $\mathrm{C}_{12}$ con los $\mathrm{H}_{\mathrm{i}} \mathrm{y} \mathrm{H}_{1}$; y la interacción de los carbonos $\mathrm{C}_{15} \mathrm{y} \mathrm{C}_{17} \operatorname{con} \operatorname{los} \mathrm{H}_{\mathrm{i}} \mathrm{y} \mathrm{H}_{1}$.

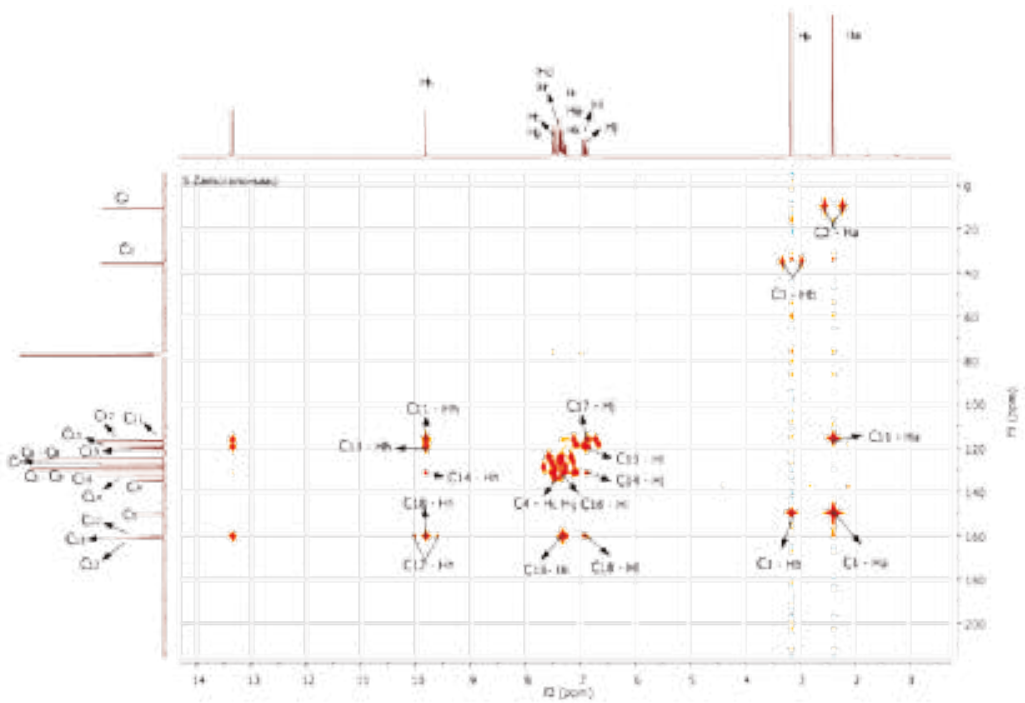

Figura 14. Espectro de RMN HMBC de la Base de Schiff 4AAP-OH 
En este espectro se puede observar la interacción del $\mathrm{C}_{11}, \mathrm{C}_{13}, \mathrm{C}_{14} \mathrm{y} \mathrm{C}_{18}$ con el $\mathrm{H}_{\mathrm{h}}$; la interacción del $\mathrm{C}_{18}$ con los $\mathrm{H}_{\mathrm{i}}$ y $\mathrm{H}_{1}$; la interacción del $\mathrm{C}_{17}$ con el $\mathrm{H}_{\mathrm{j}}$; la interacción del $\mathrm{C}_{13}$ con el $\mathrm{H}_{1}$; la interacción del $\mathrm{C}_{14}$ con el $\mathrm{H}_{\mathrm{j}}$; la interacción del $\mathrm{C}_{16}$ con el $\mathrm{H}_{\mathrm{i}}$; $\mathrm{y}$ la interacción del $\mathrm{C}_{4}$ con los $\mathrm{H}_{\mathrm{c}}$ y $\mathrm{H}_{\mathrm{g}}$. A campo alto, también se aprecian las interacción del $\mathrm{C}_{1}$ con el $\mathrm{H}_{\mathrm{a}}$ y el $\mathrm{H}_{\mathrm{b}}$, y la interacción del $\mathrm{C}_{11}$ con el $\mathrm{H}_{\mathrm{a}}$.

\section{CONCLUSIONES}

Se sintetizaron seis bases de Schiff, mediante reacciones de condensación, derivadas de la 4 aminoantipirina que contienen átomos donores de oxígeno y nitrógeno y que potencialmente pueden ser utilizadas para sintetizar compuestos de coordinación. Estos seis compuestos fueron caracterizados por técnicas de RMN bidimensional heteronuclear HSQC y HMBC, que permitió dilucidar la estructura de las citadas moléculas y observar la influencia de los diversos grupos funcionales en el entorno $\mathrm{N}-\mathrm{C}-\mathrm{C}=\mathrm{O}$.

\section{BIBLIOGRAFÍA}

1. Zamorano S, Camus J. Caracterización de bases de Schiff derivadas de 2-aminofenol, usando RMN 1D y 2D. Rev Soc Quím Perú. 2010; 76 (2):187-193.

2. Zamorano S, Camus J. Determinación de la estructura de bases de Schiff derivadas 2-aminofenol nitro y flúor sustituidas, utilizando la RMN 1D y 2D. Rev Soc Quím. Perú. 2011; 77 (1): 27-34.

3. Collins J, Dwyer FP, Lions F. Sexadentate Chelate Compounds IV. J Am Chem Soc. 1954; 74: 3134-3136.

4. Dwyer FP, Gill NS. Gyarfas EC, Lions F. Sexadentate Chelate Compounds. III1. J Am Chem Soc. 1952; 74: 4188-4193.

5. Floriani C, Calderazzo F. Oxygen adducts of Schiff's base complexes of cobalt prepared in solution. J Chem Soc A. 1969; A: 946-953.

6. Maulen R. Síntesis y caracterización de la condensación de 2-metilbenzaldehído con 4-aminoantipirina. Estudios en resonancia magnética nuclear 1D y 2D. [Tesis de Licenciatura]. Valparaíso: Universidad de Playa Ancha; 2016.

7. Martínez M. Estudio RMN 1D y 2D del producto obtenido de la condensación de 4-aminoantipirina con p-nitrobenzaldehído. [Tesis de Licenciatura]. Valparaíso: Universidad de Playa Ancha; 2012.

8. Astargo G, Encina M. Síntesis y caracterización de la condensación de la base de Schiff salicilal-4-aminoantipirina y su complejo con cobre (II). Estudios de RMN en 1D y 2D. [Tesis de Licenciatura]. Valparaíso: Universidad de Playa Ancha; 2012.

9. Aracena F. Condensación de la 4AAP con dimetilaminobenzaldehído. Estudio RMN 1D y 2D. [Tesis de Licenciatura]. Valparaíso: Universidad de Playa Ancha; 2015.

10. Hart H, Hart DJ, Craine LE. Química Orgánica. México: McGraw-Hill; 1995.

11. Cascaval A, Stoica GhZ, Berdan I. Antipyrine derivatives and process for their preparation.1993; RO 106403 B1 19930430. 
12. Moharram HH. Synthesis of some new 4-substituted antipyrines of anticipated biological activity. Pakistan J Sci Ind Res. 1985; 28(5): 300-303.

13. Shmidt EV, Prishcep TP, Chernova NA. Azomethine derivatives 4-aminoantipyrine and their anti-inflammatory activity. IzvTomsk Politekhn In-ta.1975; 250: 156-158.

14. Asiri AM, Khan SA, Marwani HM, Sharma K. Synthesis, spectroscopic and physicochemical investigations of environmentally benign heterocyclic Schiff base derivatives as antibacterial agents on the bases of in vitro and density functional theory. J Photochem Photobio B: Biology. 2013; 120: 82-89.

15. El-Ajaily MM, El-Ferjani RM, Maihub AA. Preparation and physical investigation of complexes derived from 4-dimethylaminobenzaldehyde and 4- aminoantipyrine Schiff base with Ni(II), Cu(II), Rh(III) and Pt(IV) ions. Jordan J Chem. 2007; 2(3): 287-296.

16. Al-Khamees HA, Bayomi SM, Kandil HA, El-Tajir KE. Synthesis and pharmacological screening of a new series of 3-(4-antipyryl)-2-arylthiazolidin-4-ones. Eur J Med Chem. 1990; 25(2):103-106.

17. Junaedi S, Al-Almiery AA, Kadhum AA, Mohamad AB. Inhibition effects of a synthesized novel 4-aminoantipyrine derivative on the corrosion of mild steel in hydrochloric acid solution together with quantum chemical studies. Int J Molec Sci. 2013; 14(6): 11915-11928.

18. Gupta VK, Singh AK, Kumawat LK. A turn-on fluorescent chemosensor for Zn2+ ions basedon antipyrine Schiff base. Sensor and Actuators B. 2014; 204: 507-514.

19. Sobha S, Raman N. Transition metal based biologically active compounds as selective antifungal agents. Int J Phar Bio Sci. 2012; 3(2): 116-123.

20. Baluja S, Patel A, Chanda S. Schiff bases-synthesis, characterization and antibacterial activity. Res J Pharm Bio Chem Sci. 2011; 2(4): 296-304.

21. Letunov VI. Reaction of Schiff bases, obtained from 4-aminoantipyrine and 4-amino-1,2, 4-triazole, with ketones. Zhur Org Khim. 1984; 20(1): 162-166.

22. Deshmukh P, Kumar P, Kankoriya A, Halve AK, Dixit R. 4-Aminoantipyrine: A Significant Tool for the Synthesis of Biologically Active Schiff Bases and Metal Complexes. Int J Pharm Sci Rev Res. 2015; 34(1): 162-170.

23. Raman N, Raja SJ, Sakthivel A. Transition metal complexes with Schiff-base ligands: 4-aminoantipyrine based derivatives-a review. J Coord Chem. 2009; 62(5): 691-709.

24. GT Lab. Fenoles [Internet] AQAssay; 2017. [citado el 8 de Jun. 2017]. Disponible enhttp://www.gtlab.com.ar/UserFiles/mediaManager/1/6ae042ec744b042008f4d9ac0425403fb42efdf8_ee5a0f4776a312447575d0173691b22c8bba7dcc.pdf.

25. PNT $\mathrm{N}^{\circ} 5$. Determinación colorimétrica de fenoles en agua por el método de la 4-aminoantipirina. [Internet] MNCN; 2017. [citado el 8 de Jun. 2017]. Disponible en: http://www.mncn.csic.es/docs/repositorio//es_ES//investigacion/cromatografia/ fenolfe_por_colorimetria.pdf.

26. Quiored. Elucidación estructural: Resonancia Magnética Nuclear (Desplazamiento de 13C) [Internet]. Granada: Universidad de Granada, Facultad de Ciencias Dpto. Química Orgánica; 2004. [citado el 8 de Jun. 2017]. Disponible en: http://www.ugr.es/ quiored/ espec/crmn.htm.

27. Espectroscopia RMN 2D [Internet]. Weebly; 2017. [citado el 8 de Jun. 2017]. Disponible en: http://espectroscopico.weebly.com/uploads/1/7/6/6/ 17662713/2d_nmr. pdf. 